\title{
DGAI-MITTEILUNGEN Ausschreibungen
}

DGAI Announcements

\section{Galenus-von-Pergamon-Preis 2003}

\begin{abstract}
Auch in diesem Jahr sind Wissenschaftler aus der pharmakologischen Forschung wieder aufgerufen, sich um den Galenusvon-Pergamon-Preis zu bewerben. Mit dem deutschen „Prix Galien“ zeichnet die Ärzte-Zeitung als Mitglied einer internationalen Stiftergemeinschaft alle zwei Jahre Arzneimittelforscher für herausragende wissenschaftliche Arbeiten aus, die für den Fortschritt auf dem Gebiet der Arzneimittel- und Diagnostikaforschung wegbereitend sind. Der Preis ist mit 10.000 Euro dotiert. Dem Preiskollegium, das über die Vergabe entscheidet, gehören 14 Wissenschaftler aus Medizin und Pharmakologie an.
\end{abstract}

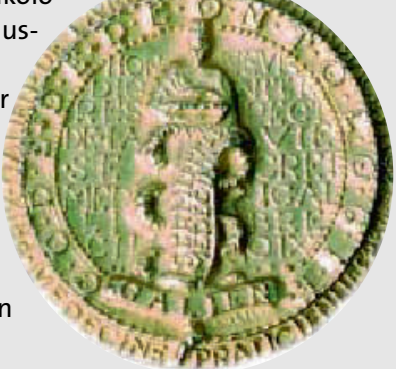

Für die Preiskategorie B bittet die Jury um Beiträge aus der klinischen und/oder experimentellen Pharmakologie. Für diese Kategorie ist Bedingung, dass die Forschungsleistung an einer Institution außerhalb der pharmazeutischen Industrie erbracht wurde. Jeder in Deutschland tätige Forscher (auch Forschungsgruppen) kann sich bewerben. Die Arbeiten dürfen bereits veröffentlicht sein, im Regelfall jedoch nicht länger als zwei Jahre vor dem Einreichungstermin. Die vorgelegten Arbeiten können bereits prämiert oder zu anderweitigen Prämierungen eingereicht worden sein. Die Bewerbungsfrist läuft bis zum 30. April 2003

\section{Sekretariat Galenus-von-Pergamon-Preis}

c/o Ärzte Zeitung Verlagsgesellschaft $\mathrm{mbH}$,

Sabine Neubert, Postfach 2002 51, 63077 Offenbach

E-Mail: sabine.neubert@aerztezeitung.de

Tel.: (o 61 02) 5 06-1 49

Internet: www.aerztezeitung.de/medizin/galenus

\section{Forschungspreis Immunmodulation}

Die Fujisawa Deutschland GmbH gibt die erstmalige Ausschreibung des Forschungspreises Immunmodulation bekannt. Der Forschungspreis bestehend aus einem Hauptpreis (10.000 Euro) und zwei Förderpreisen (je 5.000 Euro) - wird für die Entwicklung neuer Ansätze und Erkenntnisse, die zu einem verbesserten Verständnis der Immunmodulation beitragen, verliehen. Das Anliegen des Unternehmens ist es, die wissenschaftliche Forschung in den Bereichen Immunologie, Dermatologie und Allergologie zu fördern. Im jährlichen Wechsel sollen unterschiedliche Schwerpunktthemen gewürdigt werden. Das Thema 2003 heißt Ekzemforschung. Die Auswahl der Preisträger erfolgt durch eine Jury unabhängiger Experten der Dermatologie. Bewerber aus Deutschland, Österreich und der Schweiz, die Erfahrungen im klinischen Forschungsbereich von Hauterkrankungen vorweisen können, werden gebeten, bis zum 31. März 2003 folgende Unterlagen einzureichen:

— Anschreiben mit vollständiger Adresse des Bewerbers

_ Übersichtspapier (Umfang 5-7 Seiten) über bisher durchgeführte Forschungsprojekte

- Referenzliste und Kopien aller relevanten Arbeiten zum eingereichten Thema

_ Curriculum Vitae

—Zusammenfassende Darstellung der zu beurteilenden Arbeit mit einer Begründung für die Bewerbung.

Die Verleihung der Preise erfolgt am 8. Mai 2003 im Rahmen des DDG 2003 in Berlin.

\section{Forschungspreis Immunmodulation 2003}

Postfach 1103

61401 Oberursel
Forschungsprogramm Allergologie

\section{Die Landes-} stiftung BadenWürttemberg hat das

Forschungsprogramm „Allergologie“ ausgeschrieben und dafür 3,5 Millionen Euro bereitgestellt. Das Forschungsprogramm ist interdisziplinär ausgerichtet und soll grundlegende wissenschaftliche Erkenntnisse über die Mechanismen der Entstehung und Entwicklung allergischer Erkrankungen liefern. In diesem Sinne werden neue, originelle und tragfähige Ideen unterstützt, die die Voraussetzung für erfolgreichere Vorbeugung und Behandlung von Allergien in der Zukunft schaffen sollen.

Zur Teilnahme berechtigt sind Universitäten, Universitätskliniken, Fachhochschulen sowie außeruniversitäre Forschungseinrichtungen mit Sitz in Baden-Württemberg. Die Projekte sollen eine Laufzeit von drei Jahren nicht überschreiten. Anträge sind bis zum 8. Mai 2003 einzureichen.

Institut für Wissensmanagement und Innovation

Dr. Martin Grauer, Hallimaschweg 26, 70599 Stuttgart

Tel.: (07 11) 47908 o9

Fax: (07 11) 4790899

E-Mail: iwi_dr.grauer@in-win.de

Internet: www.landesstiftung-bw.de

\section{WAO Congress-XVII ICACI:}

Travel Grants

World Allergy OrganizationIAACI (WAO) will award travel grants for young scientists and fellows-intraining to attend the World Allergy Organization (WAO) Congress-XVII ICACI in Vancouver, Canada, on 7-12 September 2003. Deadline: 31 March 2003.

For application and questions regarding travel grants please contact the WAO Secretariat.

\section{WAO Secretariat}

611 East Wells Street, Milwaukee, WI 53202, USA

Phone: +1 4142761791

Fax: +1 4142763349

E-mail:congress@worldallergy.org

Website: www.worldallergy.org 